\title{
Predicting solar radiation fluxes for solar energy system applications
}

\author{
M. H. Saffaripour · M. A. Mehrabian • \\ H. Bazargan
}

Received: 7 July 2011/Revised: 29 November 2011/Accepted: 12 February 2012/Published online: 16 March 2013

(C) Islamic Azad University (IAU) 2013

\begin{abstract}
The mean daily global solar radiation flux is influenced by astronomical, climatological, geographical, geometrical, meteorological, and physical parameters. This paper deals with the study of the effects of influencing parameters on the mean daily global solar radiation flux, and also with the computation of the solar radiation flux at the surface of the earth in locations without solar radiation measurements. The reference-real data were borrowed from the Iranian Meteorological Organization. The analysis of data showed that the mean daily solar radiation flux on a horizontal surface is related to parameters such as: mean daily extraterrestrial solar radiation, average daily ratio of sunshine duration, mean daily relative humidity, mean daily maximum air temperature, mean daily maximum dew point temperature, mean daily atmospheric pressure, and sine of the solar declination angle. Multiple regression and correlation analysis were applied to predict the mean daily global solar radiation flux on a horizontal surface. The models were validated when compared with the referencemeasured data of global solar radiation flux. The results showed that the models estimate the global solar radiation flux within a narrow relative error band. The values of mean bias errors and root mean square errors were within acceptable margins. The predicted values of global solar radiation flux by this approach can be used for the design and performance estimation in solar applications. The
\end{abstract}

M. H. Saffaripour · M. A. Mehrabian ( $₫)$

Department of Mechanical Engineering,

Shahid Bahonar University of Kerman, Kerman, Iran

e-mail: ma_mehrabian@alum.mit.edu

H. Bazargan

Department of Industrial Engineering,

Shahid Bahonar University of Kerman, Kerman, Iran model can be used in areas where meteorological stations do not exist and information on solar radiation flux cannot be obtained experimentally.

Keywords Correlation coefficient · Linear regression · Mean bias error $\cdot$ Root mean square error $\cdot$ Statistical model $\cdot$ T-statistics

\section{Introduction}

Rating and sizing the solar energy systems and performance analysis of such systems requires information on solar radiation at earth's surface is available. Such information is also essential in many other applications such as crop growth models, evaporation-transpiration estimates, and building comfort conditions. There is no doubt that measured data are the best source of obtaining information on solar radiation. But, the measurement of solar radiation flux is made only in meteorological stations. Therefore, it is necessary to estimate solar radiation fluxes by theoretical models. Another approach, used for estimation purposes, involves the empirical relationships between the global solar radiation flux and more readily available influencing parameters such as sunshine duration, relative humidity, air temperature, latitude, etc. These models, however, are semi-empirical in nature and depend on spatial data of interest; that is, applying these models to conditions different from the conditions of observation is questionable at least and/or inadmissible. The parameters affecting solar radiation flux can be categorized in astronomical, climatological, geographical, meteorological, and physical factors (Ertekin and Yaldiz 1999). The astronomical factors include solar constant, world-sun distance, solar declination angle, and hour angle. The geographical factors 
include latitude, longitude, and elevation of the site. The geometrical factors include surface azimuth, surface tilt angle, solar altitude, and solar azimuth. The physical factors include scattering of air molecules, water vapor content, scattering of dust and other atmospheric constituents such as $\mathrm{O}_{2}, \mathrm{~N}_{2}, \mathrm{CO}_{2}, \mathrm{O}_{3}$, etc. The meteorological factors include effect of cloudiness, and reflection of the environs.

Many investigations were carried out to estimate the global solar radiation flux on a horizontal surface. Angstrom (1924) proposed the first empirical investigation. He correlated the global solar radiation, and the ratio of sunshine duration. Prescott (1940) and Page (1964) modified the Angstrom correlation; and the modified correlations were used to estimate the global irradiance in many countries throughout the world. However, specific models were proposed for individual countries. For example, Daneshyar (1978), Ashjaee et al. (1993), Bahadorinejad (2004) and Sabziparvar (2008) presented models for different regions of Iran; Pisimanis et al. (1987) developed a model for Greece; Ertekin et al. (1999) developed a model for Turkey; Hutchinson et al. (1984) proposed a model for Australia; Orozco (1967) developed a model for Guatemala; Feuilard et al. (1989) proposed a model for Guadeloupe; Ledanois et al. (1988) developed a model for Venezuela; Alaruri et al. (1993) proposed a model for Kuwait; Amad et al. (1983) developed a model for Iraq; Tiris et al. (1996) suggested a model for Gebze; Samuel (1991) proposed a model for Srilanka; Hawas et al. (1983) developed a model for India; Raja et al. (1989) suggested a model for Pakistan; Ezekwe et al. (1891) developed a model for Nigeria; Newland (1989) proposed a model for south China; Chuah and Donald Lee (1981) suggested a model for Malaysia; Kamel et al. (1993) developed a model for Egypt.

Furthermore, Lof et al. (1966) conducted a global study of the world distribution of solar radiation, while there were many attempts to find common models applicable anywhere in the world (Rietveld 1978) or in large regions such as Europe (Soler 1990) and humid tropical countries (Turon 1987). Singh et al. (1996) used solar declination angle and latitude to develop their empirical correlation. Reddy (1971) combined sunshine duration, air temperature, and relative humidity to estimate the global solar radiation. Sabbagh et al. (1977) related the daily global solar radiation to sunshine duration, relative humidity, maximum air temperature, altitude, and location of the place relative to water surfaces. Togrul et al. (1999) derived their equations using sunshine duration, relative humidity, air temperature, soil temperature, and the sine of the solar declination angle. Trabea et al. (2000) related the daily global solar radiation to sunshine duration, relative humidity, maximum air temperature, mean daily vapor pressure, and mean daily sea level pressure. Ertekin et al. (1999) derived their equations using sunshine duration, relative humidity, mean air temperature, mean soil temperature, solar declination angle, mean cloudiness, mean precipitation, and mean evaporation.

The purpose of this paper is to develop relationships between the global solar radiation flux and some influencing factors using the sample data. Different combinations of the mean daily extraterrestrial solar radiation intensity, average daily ratio of sunshine duration, mean daily relative humidity, mean daily maximum air temperature, mean daily maximum dew point temperature, mean daily atmospheric pressure, and the sine of the solar declination angle are used to develop the empirical equations. The coefficients in these equations were deduced, and the predicted global solar radiation values, as calculated from the model equations were compared with measured data. It is worth mentioning that solar energy in Iran is abundant, the solar energy flux incident on the land in central region of Iran (Yazd airport) has a magnitude of about 21.3 MJ/ $\mathrm{m}^{2}$ day, the sunshine duration is roughly $9 \mathrm{~h} / \mathrm{day}$, and the cloudy duration is nearly $3 \mathrm{~h} /$ day. It is expected that the applications of solar energy engineering, especially solar water heaters, become widespread in this region in the near future. Having very limited meteorological stations in this region, makes it legitimate develop correlations for estimating the desired data of global solar radiation flux at sites far from the stations. This research was carried out in the Mechanical Engineering Department, Shahid Bahonar University of Kerman, 2007-2009.

\section{Materials and methods}

\section{Selection of variables}

Multiple regression and correlation analysis are based on using one or more independent variables to obtain a more accurate prediction or to explain the variation of the dependent variable. Selecting the independent variables to predict a dependent variable is an important task; it requires lots of experience and vast knowledge on how the independent variables affect the dependent variable. When modeling solar radiation, the independent variables are selected among the influencing parameters, based on their ease of measurability, i.e., outside the meteorological station and without expensive equipment. These criteria have been accounted when selecting the independent variables to predict the solar radiation flux. Furthermore, a comprehensive literature survey has been carried out to understand how the solar radiation flux varies with the selected independent variables. A brief introduction of the independent variables used in this study is presented here. The extraterrestrial solar radiation flux (an astronomical parameter), $H_{O}\left(\mathrm{~J} / \mathrm{m}^{2}\right)$, on a horizontal surface is defined according to Duffie and Beckman (1991): 


$$
\begin{aligned}
& H_{o}=\frac{24 \times 3600 G_{0}}{\pi} \\
& \quad\left(\cos \varphi \cos \delta \sin \omega_{s}+\frac{\pi \omega_{s}}{180} \sin \varphi \sin \delta\right)
\end{aligned}
$$

$G_{0}$ is the extraterrestrial irradiance on horizontal plane, defined as:

$G_{0}=G_{s c} E_{0}=G_{s c}\left(1+0.033 \cos \frac{360 n^{*}}{365}\right)$

$G_{\text {sc }}$ is the solar constant, equal to $1,367 \mathrm{~W} / \mathrm{m}^{2}$ (ASHRAE Handbook 1985), $E_{0}$ the eccentricity correction factor of the earth's orbit, $n^{*}$ the number of the day of the year starting from January first. $\varphi$ is the latitude of the site, a geographical parameter usually known from local sources. $\omega_{\mathrm{s}}$ is the mean sunrise hour angle for the month defined as:

$\omega_{s}=\cos ^{-1}(-\tan \varphi \tan \delta)$

$\delta$ is the solar declination angle (an astronomical parameter), defined as follows:

$\delta=23.45 \sin \left(360 \frac{284+n^{*}}{365}\right)$

$N$ is the maximum possible sunshine hours from sunrise to sunset related to the mean sunrise hour angle as follows:

$N=\frac{2 \omega_{s}}{15}$

Sunshine duration ratio $(\mathrm{n} / \mathrm{N})$ is an astronomical parameter used as an independent variable in this study. This parameter takes into account the clouds; in fact $(1-n / N)$ would be the cloud duration ratio. Relative humidity, maximum air temperature, and maximum air dew point temperature are climatological parameters used as independent variables. Atmospheric pressure, a geographical parameter directly related to the elevation of the site, is used as an independent variable.

\section{Reference-real data}

Solar radiation parameters, most importantly, the hourly global solar radiation flux, are measured by the IMO at the meteorological station based in the Yazd international airport. The station is located at $31^{\circ} 54^{\prime}$ north latitude, $54^{\circ} 17^{\prime}$ east longitude and has an elevation of $1,237.2 \mathrm{~m}$ above the sea level. The data obtained from the IMO, especially the maximum air temperature, sunshine duration, and global solar radiation flux, are used as reference quantities to be compared with the model predictions. The information regarding these parameters was available in the following periods:
- Maximum air temperature, dew point temperature, and relative humidity in a 45-year period, from 1961 until 2005. Temperatures and relative humidities were measured using standard meteorological instruments approved by the world meteorological organization, Simidchiev (1986).

- Sunshine duration measured by Campbell-Stoke sunshine recorder in a 24-year period, from 1982 until 2005.

- Global solar radiation flux using pyranometer model cc-1-681 (Clipp \& Zonen, Hollands) in a 13-year period, from 1992 until 2004.

These data are used to analyze the model equations.

Statistical estimation method

The purpose of this investigation is to develop statistical models to estimate the mean daily global solar radiation flux, $\mathrm{H}$, using multiple linear regression to various parameters, such as $H_{0}, n / N, R_{h}, T_{\max }, T_{d p \text {, max }}, P$, and $\sin \delta$. To achieve this goal, the data described in the previous section were used. In linear regression, the relation between the dependent variable and the independent variables takes the following form:

$y=a+b_{i} x_{i}$

The above relation may present either a single linear regression $(i=1)$, or a multiple linear regression $(i>1)$. The dependent variable $(y)$, as well as, the independent variables $\left(x_{i}\right)$ are time-dependent. The dependent variable here is the solar radiation flux and the independent variables are the influencing parameters, all of them vary day to day from January 1 until December 31 . The regression coefficients $\left(b_{i}\right)$ depend on the site for which the measured influencing parameters are available, and the correlation is therefore accurate for that location, i.e., the functional form of the regression relation is broadly applicable, whereas the coefficient estimates are localized. Such correlations are developed based on reference data, and their predictions are compared to the measured values in each regression relation through correlation coefficient $\mathrm{R}$, defined as (Freund 2000):

$$
R=\frac{\sum_{j=1}^{n} H_{j, m} H_{j, c}-\sum_{j=1}^{n} H_{j, m} \sum_{j=1}^{n} H_{j, c}}{\sqrt{\left[n \sum_{j=1}^{n} H_{j, m}^{2}-\left(\sum_{j=1}^{n} H_{j, m}\right)^{2}\right]\left[n \sum_{j=1}^{n} H_{j, c}^{2}-\left(\sum_{j=1}^{n} H_{j, c}\right)^{2}\right]}}
$$

In addition, the deviations of the two values are shown by the relative percentage error (e), root mean square error (RMSE), mean bias error (MBE), and t-statistics. These are fundamental measures of accuracy in solar energy calculations. 
The relative percentage error is the measured-calculated deviation of an individual value, defined as (Freund 2000):

$e_{j}=\frac{H_{j, m}-H_{j, C}}{H_{j, m}} \times 100$

where $H_{j, m}$ is the $j$ th measured value and $H_{j, c}$ the $j$ th calculated value. Relative percentage errors between -10 and $+10 \%$ are considered acceptable in many engineering calculation.

The root mean square errors are defined as (Freund 2000):

$\mathrm{RMSE}=\left[\frac{1}{n} \sum_{j=1}^{n}\left(H_{j, m}-H_{j, c}\right)^{2}\right]^{1 / 2}$

where $n$ is the number of data pairs. This test provides information on the short-term performance of the correlations by allowing a term by term comparison of the actual deviation between the calculated value and the measured value. The smaller deviations are an indication of the better performance of the model. However, a few large differences in the sum can produce a significant increase in the RMSE.

The mean bias error is defined as (Freund 2000):

$\mathrm{MBE}=\frac{1}{n} \sum_{j=1}^{n}\left(H_{j, m}-H_{j, c}\right)$

This test provides information on the long-term performance. A low MBE is an indication of good model performance. A positive value gives the average amount of under-estimation in the calculated value and vice versa. A drawback of this test is that over-estimation of an individual observation will cancel under-estimation in a separate observation.

It is obvious that each test by itself may not be an adequate indicator of a model's performance. It is possible to have a large RMSE value and, at the same time, a small MBE (a large scatter about the line of perfect estimation). On the other hand, it is also possible to have a relatively small RMSE and a relatively large MBE (consistently small over-estimations or under-estimations). However, although these statistical indicators generally provide a reasonable procedure to compare models, they do not objectively indicate whether a model's estimates are statistically significant, i.e., do not significantly deviate from their measured counterparts. Therefore, an additional statistical indicator, the t-statistics, is used. The t-statistic depends on both the RMSE and MBE, so that it is more effective for determining the statistical properties. This statistical indicator allows models to be compared and at the same time indicates whether or not a model's estimates are statistically significant at a particular confidence level. The t-statistic is defined as (Almorox et al. 2005):

$t=\left[\frac{(n-1) \mathrm{MBE}^{2}}{\mathrm{RMSE}^{2}-\mathrm{MBE}^{2}}\right]^{1 / 2}$

A smaller $t$-value is an indication of the better performance of the model. To determine whether a model's estimates are statistically significant, one simply has to determine a critical $t$-value from standard statistical tables, i.e., $t_{\alpha / 2, n-1}$ at $\alpha$ level of significance and $(n-1)$ degrees of freedom. For the model's estimate to be judged statistically significant at the $1-\alpha$ confidence level, the calculated $t$-value must be less than the critical $t$-value.

\section{Results and discussion}

Multiple linear regression of seven parameters $H_{0}$, sin $\delta$, $n / N, R_{h}, T_{\max }, T_{d p, \max }, P$ in different combinations gave many different correlations to estimate the global solar radiation fluxes. They were processed and analyzed using an in-house computer program to obtain the correlations and the regression coefficients $a$, and $b_{i}$ in Eq. 6. A least square regression analysis was used to obtain these constants.

The computer program selects the equations with the highest correlation coefficients and the lowest t-statistics among the possible regression relations with one to seven independent variables. The independent variables in the selected regression relations to meet the above qualifications are:

- One-variable relations using $\sin \delta$ or $\mathrm{H}_{0}$

- Two variable relations using $\sin \delta, n / N$ or $n / N, H_{0}$

- Three variable relations using $n / N, H_{0}, R_{h}$ or $n / N, H_{0}, T_{\max }$

- Four variable relations using $n / N, H_{0}, R_{h}, P$ or $n / N, H_{0}, T_{\max }, T_{d p, \max }$

- Five variable relations using $\sin \delta, n / N, T_{\max }$, $T_{d p, \max }, H_{0}$ or $n / N, H_{0}, T_{\max }, T_{d p, \max }, R_{h}$

- Six variable relations using $H_{0}, \sin \delta, n / N, R_{h}$, $T_{\max }, T_{d p, \max }$ and $H_{0}, n / N, R_{h}, T_{d p, \max }, P, T_{\max }$

- Seven-variable relations using $H_{0}, \sin \delta, n / N, R_{h}$, $T_{\max }, T_{d p, \max }, P$

The regression relations based on the above independent variables are listed in Table 1.

The values of mean daily global solar radiation fluxes estimated using the above regression correlations were compared with the corresponding reference-measured values. The results concerning the seven-variable correlation are illustrated in Fig. 1. The figure shows that the agreement between the measured and estimated values is remarkable. This is why the higher order regression relations are not necessary. 
A dummy variable investigation is carried out with the independent variables being introduced on a monthly basis. The resulting regression relation when seven-variables are involved is:

$$
\begin{aligned}
& \bar{H}=13.3170-0.5155 \sin \bar{\delta}+0.7441 \bar{H}_{0}+23.0620 \bar{n} / \bar{N} \\
& +0.0142 \bar{R}_{h}-0.1188 \bar{T}_{\max }+0.0244 \bar{T}_{d p, \max }-0.0335 \bar{P}
\end{aligned}
$$

The values of $R$ and $t$ for the above equation are 0.993 and 0 , respectively. The predicted global solar radiation fluxes by Eqs. 24 and 25 are compared with the measured data in Fig. 1. This comparison is based on measured data averaged over a 13-year period. The figure shows that Eq. 25 (dummy variable) gives more accurate results.

The data resulting in Eqs. 12-24 are the mean daily values over a 13 -year period. In order to examine whether or not the coefficients $b_{i}$ are constant over time, the data in a 3-year period were used to develop a regression relation based on seven independent variables. The new equation turned out to be:

$$
\begin{gathered}
H=12.66+7.71 \sin \delta+0.43 H_{0}+10.39 n / N \\
+0.02 R_{h}+0.09 T_{\max }-0.16 T_{d p, \max }-0.02 p
\end{gathered}
$$

Comparing Eq. 26 with Eq. 24 shows that regression coefficients $a$, and $b_{i}$ vary over time. The values of $R$ and $t$ for the above equation are 0.9808 and 0 , respectively. The global solar radiation fluxes predicted by Eqs. 24 and 26 are compared with the measured data in Fig. 2. This comparison is based on the reference-measured data averaged over a 3-year period.

\section{Daily/monthly data collection}

The regression coefficients in Eqs. 12-24 were calculated based on data of influencing parameters averaged on a daily basis over a 13-year period. The regression coefficients in Eq. 25 were calculated based on data of influencing parameters averaged on a monthly basis over a 13-year period. The seven-variable models (Eqs. 24 and 25) are compared with each other and also with measured data of global solar radiation flux averaged on a daily basis over a 13-year period in Fig. 1. This comparison shows that the seven-variable model based on data of influencing parameters averaged on a monthly basis gives more accurate results as compared with the seven-variable model based on data of influencing parameters averaged on a daily basis.

Period of data collection

The regression coefficients in Eq. 26 were calculated based on data of influencing parameters averaged on a daily basis over a 3-year period. The seven-variable models (Eqs. 24 and 26) are compared with each other and also with measured data of global solar radiation flux averaged on a daily basis over a 3-year period in Fig. 2. This comparison shows that the regression coefficients $a$, and $b_{i}$ in Eq. 6 are time dependent, i.e., they vary with the period of the data over which the regression coefficients were calculated.

\section{Statistical criteria}

The t-statistic was reported for each regression correlation to evaluate the accuracy of the estimated data. This is because the $t$ test is an effective criterion for determining the statistical properties. The critical $t$-value for 11 degrees of freedom and 0.025 levels of significance is extracted from standard statistical tables (Freund 2000) as 2.201. This value is the same for all regression relations in Table 1. Higher $t$-values than the critical $t$-value show that the relation has no statistical significance. The reason why $R^{2}$ has not been used as a statistical indicator in this study is because there are several misconceptions about $R^{2}$. In general, $R^{2}$ does not measure the magnitude of the slope of the regression line. A large value of $R^{2}$ does not imply a steep slope. Furthermore, $R^{2}$ does not measure the appropriateness of the model, since it can be artificially inflated by adding higher order polynomial terms to the model. Even if the variables are related in a nonlinear fashion, $R^{2}$ will often be large (Montgomery 1994).

\section{Model selection}

Generally speaking, Eq. 24 shows the best results among the relations developed in this study. Since this relation gives the smallest values of the statistical indicator, it is considered as the best relation for estimating the global solar radiation flux with very small error. On the other hand, however, seven different parameters are involved if one decides to use this model for predicting the global solar radiation flux. It is of great concern to realize that selecting seven independent variables among the influencing parameters to predict the global solar radiation flux is very idealistic. There are occasions when very high accuracy is not the major concern, and the user decides to make his predictions based on models with lower number of independent variables.

Table 1 shows even when only one independent variable $(\sin \delta)$ is selected, the correlation coefficient is 0.9383 and the t-statistic is 0.0209 . That means the one-variable regression correlation (Eq. 12) predicts the global solar radiation flux with an accuracy which is satisfactory in most engineering applications. The diversity of regression relations gives the engineer a broad freedom of choices. 
Table 1 Linear regression relations using 1, 2, 3, 4, 5, 6, and 7 variables with the highest correlation coefficients

\begin{tabular}{|c|c|c|c|}
\hline Selected variables & Regression relationship & $\begin{array}{l}\mathrm{R} \\
\mathrm{t}\end{array}$ & Eq. No \\
\hline \multirow[t]{2}{*}{$\sin \delta$} & $H=21.3360+20.9900 \sin \delta$ & 0.93828 & Eq. 12 \\
\hline & & 0.0209 & \\
\hline \multirow[t]{2}{*}{$H_{O}$} & $H=-1.1916+0.7285 H_{0}$ & 0.93857 & Eq. 13 \\
\hline & & 0.0133 & \\
\hline \multirow[t]{2}{*}{$\sin \delta, n / N$} & $H=10.7980+18.7300 \sin \delta+14.2170 n / N$ & 0.96026 & Eq. 14 \\
\hline & & 0.039 & \\
\hline \multirow[t]{2}{*}{$n / N, H_{0}$} & $H=-9.6587+14.7450 n / N+0.6489 H_{0}$ & 0.96243 & Eq. 15 \\
\hline & & 0.0363 & \\
\hline \multirow[t]{2}{*}{$n / N, H_{0}, R_{\mathrm{h}}$} & $H=-7.5046+13.5340 n / N+0.6282 H_{0}+0.0205 R_{h}$ & 0.96254 & Eq. 16 \\
\hline & & 0.0377 & \\
\hline \multirow[t]{2}{*}{$\mathrm{n} / N, H_{0}, \mathrm{~T}_{\max }$} & $H=-8.5725+13.2110 n / N+0.6166 H_{0}+0.0394 T_{\max }$ & 0.96258 & Eq. 17 \\
\hline & & 0.043 & \\
\hline \multirow[t]{2}{*}{$n / i t N, H_{0}, R_{\mathrm{h}}, P$} & $H=50.7690+13.0860 n / N+0.5965 H_{0}-0.0267 R_{\mathrm{h}}-0.0648 P$ & 0.96268 & Eq. 18 \\
\hline & & 0.0382 & \\
\hline \multirow[t]{2}{*}{$n / N, H_{0}, T_{\max }, T_{d p, \max }$} & $H=-9.7002+12.2200 n / N+0.6347 H_{0}-0.0765 T_{\max }-0.1754 T_{\mathrm{dp}, \max }$ & 0.96288 & Eq. 19 \\
\hline & & 0.0359 & \\
\hline \multirow[t]{2}{*}{$\sin \delta, n / N, T_{\max }, T_{\mathrm{dp}, \max }, H_{0}$} & $H=-5.1669+4.6974 \sin \delta+12.0540 n / N-04.859 H_{0}+0.0799 T_{\max }-0.231 T_{\mathrm{dp}, \max }$ & 0.96301 & Eq. 20 \\
\hline & & 0.038 & \\
\hline \multirow[t]{2}{*}{$n / N, H_{0}, T_{\max }, T_{\mathrm{dp}, \max }, R_{\mathrm{h}}$} & $H=-15.0560+12.0130 n / N+0.6430 H_{0}+0.1905 T_{\max }+0.0660 R_{\mathrm{h}}-0.3033 T_{\mathrm{dp}, \max }$ & 0.96309 & Eq. 21 \\
\hline & & 0.0403 & \\
\hline \multirow[t]{2}{*}{$H_{0}, \sin \delta, n / N, R_{\mathrm{h}}, T_{\max }, T_{\mathrm{dp}, \max }$} & $H=-11.9620+2.1248 \sin \delta+11.9780 n / N+0.5741 H_{0}+0.1698 T_{\max }+0.0531 R_{h}-0.3036 T_{\mathrm{dp}, \max }$ & 0.96311 & Eq. 22 \\
\hline & & 0.0404 & \\
\hline \multirow[t]{2}{*}{$H_{0}, n / N, R_{\mathrm{h}}, T_{\mathrm{dp}, \max }, P, T_{\max }$} & $H=-63.8510+11.8390 n / N+0.6658 H_{0}+0.2536 T_{\max }+0.1004 R_{\mathrm{h}}-0.3735 T_{\mathrm{dp}, \max }+0.0518 P$ & 0.96313 & Eq. 23 \\
\hline & & 0.0433 & \\
\hline \multirow[t]{2}{*}{$H_{0}, \sin \delta, n / N, R_{\mathrm{h}}, T_{\max }, T_{\mathrm{dp}, \max }, P$} & $H=-61.8680+2.2328 \sin \delta+0.5940 H_{0}+11.7980 n / N+0.0878 R_{\mathrm{h}}+0.2335 T_{\max }-0.3756 T_{\mathrm{dp}, \max }+0.0531 P$ & 0.96315 & Eq. 24 \\
\hline & & 0.0435 & \\
\hline
\end{tabular}

Knowing only one astronomical parameter of the site $(\sin \delta)$ makes him capable of estimating the global solar radiation flux. Of course, when more independent variables are available, more accurate results are predicted.

\section{Independent variables}

The fact worth to mention is that the parameters used to develop the models are not totally independent; for example, air temperature is not independent of solar declination angle and so on. It should, however, be noticed that the parameters have been measured independently, and no analytical relationship between the parameters has been used in developing the models. From this point of view, the parameters have been treated as being independent.

\section{Conclusion}

Reference-real meteorological data on several solar parameters were obtained, investigated and analyzed.
Many types of correlations were tested against experimental results and relations having the highest correlation coefficients $(R)$ were selected. The correlation and regression coefficients for each selected relation were calculated. New multiple regression correlations that give accurate estimates of mean daily global solar radiation flux on a horizontal surface were suggested. The following conclusions were drawn:

- The values of the correlation coefficient vary between $93.83 \%$ for the correlation with one-variable and $96.31 \%$ for the correlation with seven-variables, and the relative error did not exceed $4.38 \%$.

- The accuracy of the estimation is higher for the correlations involving more independent variables. The seven-variable relation has the values of MBE $\left(0.0052 \mathrm{MJ} / \mathrm{m}^{2}\right.$ day), and RMSE $\left(0.3947 \mathrm{MJ} / \mathrm{m}^{2}\right.$ day) within the acceptable ranges. This indicates that the model presents valid estimates of the mean global solar radiation flux.

- Until solar radiation can be measured in more stations empirically, the model can be applied to any site in central 
Fig. 1 Comparison of measured and estimated values using Eq. 14 and Eq. 25 (The measured data are averaged over a 13 year period)
Fig. 2 Comparison of measured and estimated values using Eqs. 24 and 26 (The measured data are averaged over a 3-year period)
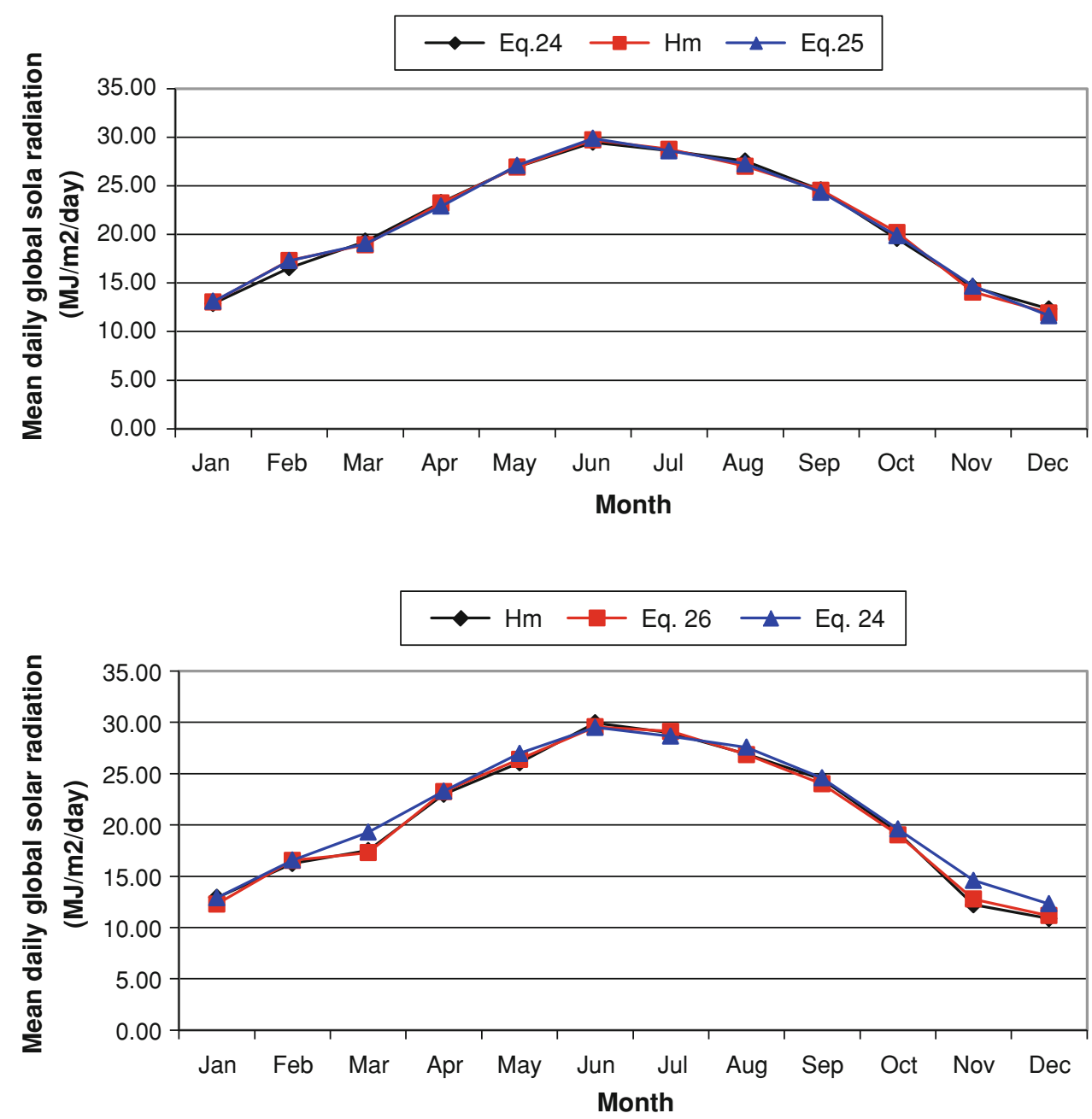

region of Iran. The only requirement for this application is that one measures or calculates at least one of the influencing parameters discussed in this paper. This can be accomplished at any remote site in the arid region of the country. The global solar radiation flux values predicted by this approach can be used in the design and performance estimation of solar energy systems, which are gaining increasing attention in this region.

Acknowledgments The authors would like to express their gratitude to the Iranian Meteorological Organization (IMO) for their sincere cooperation in providing files and documents available in their archive containing meteorological information regarding Yazd airport station. Without this information this research would have not been successful.

\section{Nomenclature}

a Regression coefficient, Eq. 6

$b \quad$ Regression coefficient, Eq. 6

c Subscript for calculated

$e \quad$ Relative percentage error

$E_{0} \quad$ Eccentricity correction factor
$G_{0} \quad$ Extraterrestrial irradiance

$G_{\mathrm{sc}} \quad$ Extraterrestrial solar constant

$H_{\mathrm{o}} \quad$ Mean daily extraterrestrial radiation

$H \quad$ Mean daily global radiation flux

IMO Iranian Meteorological Organization

$i \quad$ Counter for dependent variables

$j \quad$ Counter for number of data pairs

$m \quad$ Subscript for measured

$M B E \quad$ Mean bias error

$N \quad$ Potential astronomical sunshine hours

$n \quad$ Hours of measured sunshine

$n \quad$ Number of data pairs, Eqs. 8, 10 and 11

$n-1 \quad$ Degrees of freedom, Eq. 12

$n^{*} \quad$ Day of year

$P \quad$ Mean daily atmospheric pressure

$R \quad$ Coefficient of correlation

$R_{\mathrm{h}} \quad$ Mean daily relative humidity

RMSE Root mean square error

$T_{\mathrm{dp}, \max }$ Mean daily maximum dew point temperature

$T_{\max } \quad$ Mean daily maximum air temperature

$t \quad$ Test statistic 
$x \quad$ Dependent variable, Eq. 6

$y \quad$ Independent variable, Eq. 6

$\varphi \quad$ Latitude of the site

$\alpha \quad$ Level of significance

$\delta \quad$ Solar declination angle

$\omega_{\mathrm{s}} \quad$ Mean sunrise hour angle

\section{References}

Alaruri SD, Amer MF (1993) Empirical regression models for weather data measured in Kuwait during the years 1985, 1986, and 1987. Sol Energy 50:229-233

Almorox J, Benito M, Hontaria C (2005) Estimation of monthly Angstrom-Prescott equation coefficients from measured daily data. Renewable Energy 30:931-936 (6 pages)

Amad I, Al-Hamdani N, Ibrahim K (1983) Solar radiation maps for Iraq. Sol Energy 31:29-44

Angstrom A (1924) Solar and terrestrial radiation. Quart J Roy Meteor Soc 50:121-126

Ashjaee M, Roomina MR, Ghafouri-Azar R (1993) Estimating direct, diffuse, and global solar radiation for various cities in Iran by two methods and their comparison with the measured data". Sol Energy 50(5):441-446

ASHRAE Handbook, Fundamental, SI edition (1985) American Society of Heating, Refrigerating, and Air-Conditioning Engineers

Bahadorinejad M, Mirhosseini SA (2004) Clearness index data for various cities in Iran, Presented at the Third Conference on Optimization of Fuel Consumption in Building, Persian Volume, pp 603-619

Chuah GS, Donald Lee SL (1981) Solar radiation estimates in Malaysia. Sol Energy 26:33-40

Daneshyar M (1978) Solar radiation statistics for Iran. Sol Energy 21:345-349

Duffie JA, Beckman WA (1991) Solar engineering of thermal processes. Wiley, New York

Ertekin C, Yaldiz O (1999) Estimation of monthly average daily global radiation on horizontal surface for Antalya, Turkey. Renewable Energy 17:95-102 (8 pages)

Ezekwe CI, Ezeilo Clifford CO (1891) Measured solar radiation in a Nigerian environment compared with predicted data. Sol Energy 26:181-186

Feuilard T, Aillion JM (1989) Relationship between global solar irradiation and sunshine duration in Guadeloupe. Sol Energy 43:356-361

Freund JE (2000) Mathematical Statistics. Prentice Hall of India

Hawas MM, Muneer T (1983) Correlation between global radiation and sunshine data for india. Sol Energy 30:289-290

Hutchinson MF, Booth TH, McMahoon JP, Nix HA (1984) Estimating monthly mean value of daily total solar radiation for Australia. Sol Energy 32:277-290

Kamel MA, Shalaby SA, Mostafa SS (1993) Solar radiation over Egypt: comparison of predicated and measured meteorological data. Sol Energy 50:463-467
Ledanois JM, Prieto A (1988) A new solar map of Venezuela. Solar Energy 41:115-126

Lof GOG, Duffie JA, Smith CO (1966) World distribution of solar radiation. Solar Energy 10:27-37 (11 pages)

Montgomery DC, Montgomery GCR (1994) Applied statistics and probability for engineers. Wiley, New York

Newland FJ (1989) Study of solar radiation models for the coastal region of South China. Sol Energy 43:227-235

Orozco EB (1967) Guatemalan solar map. Solar Wind Technology $4: 381-388$

Page JK (1964) The estimation of monthly mean values of daily total short wave radiation on vertical and inclined surfaces from sunshine records for latitude $40^{\circ} \mathrm{N}-40^{\circ} \mathrm{S}$. In: Proceedings of UN conference on new sources of energy

Pisimanis D, Notaridou V (1987) Estimating direct, diffuse and global solar radiation on an arbitrary inclined plane in Greece. Sol Energy 39:159-172

Prescott JA (1940) Evaporation from a water surface in relation to solar radiation. Trans Roy Soc South Austr 64:114-125

Raja IA, Twidell JW (1989) Distribution of global insolation over Pakistan. Sol Energy 43:335-357

Reddy SJ (1971) An empirical method for estimation of the total solar radiation. Sol Energy 13:289

Rietveld MR (1978) A new method for estimating the regression coefficients in the formula relating solar radiation to sunshine. Agr Meteorlol 19:243-252 (10 pages)

Sabbagh JA, Sayigh AA (1977) El-Salam Ma. Estimation of the total solar radiation from meteorological data. Sol Energy 19:307-311 (5 pages)

Sabziparvar AA (2008) A simple formula for estimating global solar radiation in central arid deserts of Iran". Renewable Energy 33(5):1002-1010

Samuel TDMA (1991) Estimation of global radiation for Srilanka. Sol Energy 47:333-337

Simidchiev DA (1986) Compendium of lecture notes on meteorological instruments for training class III and class IV meteorological personnel, World Meteorological Organization, Vol 1, No. 622. Geneva

Singh OP, Srivastava SK, Guar A (1996) Empirical relationship to estimate global radiation from hours of sunshine. Energy Convers Manage 37:501-504 (4 pages)

Soler A (1990) Statistical comparison for 77 European stations of sunshine based models. Sol Energy 45:365-370 (6 pages)

Tiris M, Tiris C, Ture E (1996) Correlations of monthly average daily global, diffuse and beam radiation with hours of bright sunshine in Gebze. Energ Convers Manag 37:1417-1421

Togrul IT, Onat E (1999) A study for estimating solar radiation in Elazing using geographical and meteorological data. Energ Convers Manag 40:1577-1584 (8 pages)

Trabea AA, Shaltout MAM (2000) Correlation of global solar radiation with meteorological parameters over Egypt. Renewable Energy 21:297-308 (12 pages)

Turon SM (1987) The relationship between total irradiation and sunshine duration in the humid tropics. Sol Energy 38:353-354 (2 pages) 